\title{
Rhabdomyolysis with simvastatin
}

\author{
Brijesh R Patel, ${ }^{1}$ Maitrayee Choudhury ${ }^{2}$ \\ ${ }^{1}$ Colchester General Hospital, Colchester, UK; \\ ${ }^{2}$ Queen's Hospital, Romford, UK \\ Correspondence to Brijesh R Patel, brijeshrpatel@doctors.org.uk
}

\section{SUMMARY}

As 3-hydroxy-3-methylglutaryl coenzyme A (HMG-CoA) reductase inhibitors ('statins') are being increasingly prescribed for the prevention of cardiovascular disease, this case report describes adverse effects of statin therapy which can sometimes be fatal. It highlights the need for physicians to be aware of individual patient risk factors predisposing to statin induced myopathy. It also highlights the need for further research into cholesterol lowering drugs which do not have such side effects. When prescribing statins for patients, we must also explain potential side effects following initiation of therapy and with dose titrations.

\section{BACKGROUND}

Rhabdomyolysis associated with statin therapy has an incidence of 3.4 per 100000 patient-years of treatment and is well publicised to be secondary to pharmacological interactions. ${ }^{12}$ It results from the breakdown of striated muscle fibres leading to the release of myoglobin into the blood stream with an associated 10-fold elevation of creatine kinase ${ }^{3}$ above the normal limit. This can lead to the life-threatening complications of acute renal failure, disseminated intravascular coagulation and hyperkalaemia. The mean time of onset of myopathy side effects is approximately 6 months after initiation of treatment, although symptoms can occur at any time during statin therapy. ${ }^{4}$

\section{CASE PRESENTATION}

A 74-year-old Asian woman of normal weight was admitted to the coronary care unit for cardiac monitoring having developed sick sinus syndrome requiring pacemaker implantation. The patient had a history of hypertension and raised cholesterol which had been treated with simvastatin at a dose of $20 \mathrm{mg}$ a day for 1 month. Three weeks prior to her admission the simvastatin dose was increased to $40 \mathrm{mg}$. Following this, the patient began passing dark coloured urine, and her creatinine was noted to be raised (154 mmol/l) on admission with a previous creatinine clearance of $52.1 \mathrm{ml} / \mathrm{min}$.

Over the next 2 days the patient's creatinine rose to 353 $\mathrm{mmol} / \mathrm{l}$ and she began to complain of muscular thigh pain. A drug induced myositis was considered and blood test were carried out (table 1).

\section{TREATMENT}

The patient was commenced on crystalloid fluids initially at a rate of $1 \mathrm{l} / \mathrm{h}$ and received sodium bicarbonate therapy. Her simvastatin was discontinued.

\section{OUTCOME AND FOLLOW-UP}

Electrolytes, urea, creatinine and liver function test were monitored regularly (figure 1). Following 2 weeks of intravenous fluid therapy, the serum creatinine fell to $251 \mathrm{mmol} / \mathrm{l}$ and the creatine kinase was $487 \mathrm{mmol} / \mathrm{l}$. The patient had a pacemaker device implanted and was discharged with outpatient follow-up. Subsequent blood test results showed a normal creatinine clearance.

\section{DISCUSSION}

\section{Statin therapy and rhabdomyolysis}

Statins reduce melanovate synthesis, a crucial precursor to endogenous liver production of cholesterol, and are currently the most effective low-density lipoprotein (LDL) treatment. They are being increasingly used as part of primary and secondary 5 prevention of cardiovascular disease. However, statin therapy has many side effects (box 1), ${ }^{6}$ the most serious being rhabdomyolysis. Muscle breakdown results in myoglobin being released, which is oxidised creating myoglobin casts that cause renal tubular obstruction and acute renal failure; $10 \%$ of such cases are fatal. ${ }^{7}$

Presentation is usually benign, with many patients ignoring the early symptoms of myopathy. ${ }^{8}$ Classically, patients present with muscle pain, weakness and dark urine. There is little evidence to support a specific time frame in which rhabdomyolysis is likely to occur after starting statins or increasing the dose, however the mean time of onset for cases involving atorvastatin and simvastatin is approximately 1 year after beginning therapy. ${ }^{9}$

The mechanism of statin induced rhabdomyolysis is poorly understood. Theoretically, decreased melanovate

\section{Box 1 Side effects of statin therapy 6}

- Headache

- Liver impairment

- Paraesthesia

- Gastrointestinal disturbance

- Myopathy/myalgia

- Rhabdomyolysis

- Rash

Hypersensitivity (rare) 


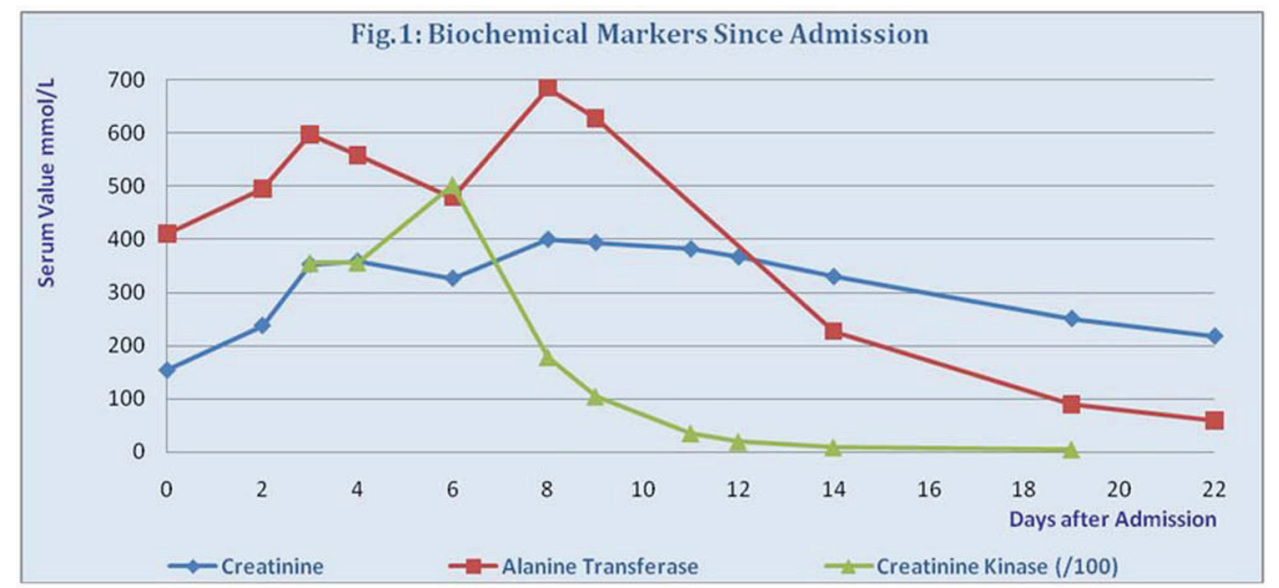

Figure 1 The patient's biochemical markers after admission.

Table 1 The patient's biochemistry results (normal values) on the third day after admission

\begin{tabular}{ll} 
Sodium $136(135-145) \mathrm{mmol} / \mathrm{l}$ & Bilirubin $18(0-17) \mathrm{mmol} / \mathrm{l}$ \\
Potassium $4.7(3.5-5.0) \mathrm{mmol} / \mathrm{l}$ & Alkaline phosphatase $55(42-128) \mathrm{IU} / \mathrm{I}$ \\
Creatinine $353(58-96) \mathrm{mmol} / \mathrm{l}$ & Alanine transferase $597(0-40) \mathrm{IU} / \mathrm{l}$ \\
Urea $29.5(1.7-8.3) \mathrm{mmol} / \mathrm{l}$ & Creatinine clearance $11.6(>50) \mathrm{ml} / \mathrm{min}$ \\
Creatinine kinase $35456(0-145) \mathrm{IU} / \mathrm{l}$ & \\
\hline
\end{tabular}

production may disrupt small regulatory proteins required for myocyte cell membrane maintenance, but in vitro models fail to demonstrate myotoxicity. ${ }^{10}$ Reduced serum ubiquinone (coenzyme $\mathrm{Q}_{10}$ ), another product of the HMGCoA reductase/melanovate pathway that is responsible for electron transfer in mitochondria, has also been suggested as a possible aetiology. More recent evidence suggests that suppressed HMG-CoA reductase/melanovate pathway dependant protein synthesis, in particular of farnesol and geranylgeraniol, is involved in statin induced apoptosis, leading to rhabdomyolysis ${ }^{8}$ and there also may be some involvement causing impaired fat oxidation. ${ }^{11}$

\section{Risk factors}

The risk of statin induced myotoxicity is multifactorial, and varies with both the pharmacological properties of the individual statins and individual patient characteristics. Transport of statins into the liver is an active process via the organic polypeptide transporter 2 found on hepatic cells. ${ }^{12}$ In myocytes the transport is dependent on passive diffusion, thus serum concentration (and dosage) has been shown to increase the risk of rhabdomyolysis ${ }^{13}$ although in a non-linear fashion. ${ }^{14}$

Factors affecting volume distribution and statin metabolism such as increased age and renal and hepatic dysfunction, all increase risk. ${ }^{15}$ Recent surgery ${ }^{16}$ or extremely heavy exercise ${ }^{17}$ have both been shown to increase the risk of statin induced rhabdomyolysis and discontinuation of statins before such planned events has been advocated. ${ }^{8}$ Other co-morbidities including hypothyroidism and diabetes also increase the risk of statin induced rhabdomyolysis. ${ }^{3}$

The majority of statins are metabolised in the liver by the cytochrome P450 (CYP) enzymes. Simvastatin, atorvastatin and lovastatin are metabolised by the 3A4 subgroup
Table 2 Co-prescribed drugs most commonly causing rhabdomyolysis with statins ${ }^{19}$

\begin{tabular}{ll}
\hline Fibrates & $38 \%$ \\
Digoxin & $5 \%$ \\
Warfarin & $4 \%$ \\
Macrolides & $3 \%$ \\
Cyclosporin & $2 \%$ \\
Mibefradil & $2 \%$ \\
Antifungals & $1 \%$ \\
\hline
\end{tabular}

and have shown have a slightly increased risk of rhabdomyolysis (4.2 per 100000 patient-years). Medications that inhibit CYP-3A4 have been shown to increase the risk of rhabdomyolysis when given simultaneously with a CYP-3A4 metabolised statin. ${ }^{13}$ CYP-3A4 activity can vary 10 -fold ${ }^{18}$ between patients, believed to be a result of genetic polymorphism. ${ }^{19}$

However, it should be noted that up to $60 \%$ of statin prescriptions have an interacting drug co-prescribed, but only $3 \%$ result in adverse reactions (the co-prescribed drugs most commonly causing rhabdomyolysis are listed in table 2). ${ }^{20}$ Fluvastatin (metabolised by the CYP-subgroup 2A9) and pravastatin (which is water soluble, and hence not metabolised by CYP enzymes) have been quoted to have an approximately fourfold lower incidence of rhabdomyolysis, 7 but are less effective at lowering LDL cholesterol. ${ }^{21}$ There is also evidence that statins are metabolised by the glucuronidation pathway and that MRP2 inhibitors such as gemfibrozil can increase the risk of rhabdomyolysis 10 -fold. ${ }^{22}$

Although no direct link between ethnicity and risk of statin induced myotoxicity has been shown, studies have demonstrated increased serum statin levels in those of Asian origin ${ }^{23}$ as was the case with our patient. In addition female sex, advancing age, low body mass index and concurrent illness have all been shown to be high risk for statin induced myopathy. ${ }^{24}$ All these factors place the patient described in this case report at higher risk for statin induced myopathy, with no potential drug interactions or predisposing co-morbidities. Recent studies have identified common variants in the SLCO1B1 gene involved in hepatic uptake of statins that are linked to statin induced myopathy. ${ }^{25}$ 


\section{Management of statin induced rhabdomyolysis}

Rhabdomyolysis is defined as an elevated creatine kinase 10 times the upper limit of normal (>1450 IU/1), and if found statin therapy should be terminated immediately. The principles of acute management of rhabdomyolysis are to treat the underlying cause and diuresis through aggressive rehydration aimed at diluting myoglobin in the renal tubules. ${ }^{14}$ An alkaline diuresis can be induced with bicarbonates to stabilise oxidised myoglobin that is responsible for renal tissue injury, and is most effective in the first 8 h. ${ }^{26}$ However, an alkaline diuresis can be achieved by fluid therapy alone, with some papers quoting initial infusion rates of up to $1.5 \mathrm{l} / \mathrm{h}$ of normal saline; once the patient is haemodynamically stable this can be reduced to $300-500$ $\mathrm{ml} / \mathrm{h}$ and intravenous infusion should be continued until creatine kinase values are below 1000 IU/1. ${ }^{27}$

Severe cases of rhabdomyolysis may require haemofiltration or dialysis. In addition, mannitol can be used to facilitate renal perfusion and excretion of myoglobin. ${ }^{14}$ Furosemide can precipitate further myoglobin accumulation in the renal tubule and calcium replacement with hypophosphataemia can induce further muscle necrosis, so both should be avoided. ${ }^{26}$ Unfortunately there few randomised controlled trials concerning therapies additional to intravenous fluid therapy in rhabdomyolysis and thus no concrete indications for bicarbonate or mannitol therapy. However, the majority of patient treated early with intravenous fluids regain normal renal function. ${ }^{28}$

Urea and electrolytes should be monitored, in particular potassium for hyperkalaemia and in severe cases cardioprotective calcium is indicated. ${ }^{26}$ It should be noted that bicarbonate therapy may cause an intracellular shift of potassium leading to serum hypokalaemia. ${ }^{14}$

Despite various case reports, there is little evidence to suggest that statin therapy carries an increased risk of hepatotoxicity in patients with no pre-existing liver disease. Studies show that the rare incidences are no more common than in the general population. ${ }^{7}$ It has been suggested in cases of suspected statin induced rhabdomyolysis, that raised aspartate aminotransferase is of assumed muscle origin if the alanine aminotransferase (ALT) is normal. ${ }^{29}$ In the case presented here, raised ALT levels indicated some degree of hepatic impairment, more likely secondary to increased serum simvastatin concentration outside the therapeutic range. ${ }^{14}$

With regards to statin therapy, prevention via patient education is essential. Patients should be aware of the side effects of myopathy and report any myalgia or darkening of urine to their physician. ${ }^{6}$ Similarly, physicians should use the lowest dose of statin required to meet target cholesterol levels and avoid using them with drugs known to potentiate myotoxicity unless necessary, although there is no absolute contraindication. ${ }^{3}$

there are reports of patients after acute treatment of rhabdomyolysis being restarted on lower doses of the offending statin ${ }^{30}$ or an alternative statin, ${ }^{31}$ however there are few data allow a confident conclusion. ${ }^{8}$ It has also been suggested that many patients restarted on statins again experience symptoms of myopathy. ${ }^{8}$ Despite the fact that there is some evidence that hydrophilic statins may have less myotoxic side effects, there is still some controversy over this issue with case reports of patients who after simvastatin induced rhabdomyolysis were switched to pravastatin and redeveloped symptoms. ${ }^{14}$ There is also evidence to suggest that patients who have had rhabdomyolysis secondary to statin therapy should be investigated for a predisposing metabolic muscle deficit. ${ }^{32}$

Alternative lipid lowering therapies include fibrates, nicotinic acids and eztimibe, which although not contraindicated, are discouraged after statin induced rhabdomyolysis by specialist statin myopathy clinic studies. ${ }^{14}$ Both fibrates and nicotinic acids should be used cautiously in renal failure, which may be residual after an acute episode of statin induced rhabdomyolysis. Eztimide, which inhibits intestinal absorption of cholesterol, has on very rare occasions caused a raised creatine kinase level, myopathy and rhabdomyolysis.

An aggressive low fat diet therapy is the mainstay recommendation for the management of hyperlipidaemia after statin induced rhabdomyolysis. The use of resins, which are known to have minimum effect on fatty acid oxidation, has been examined in trials in statin myopathy clinics, and so far are tolerated well. ${ }^{14}$

\section{Learning points}

- Statin induced rhabdomyolysis can have serious consequences with high mortality.

- Patients should be informed of the risks and warning symptoms.

- Clinicians should screen for risk factors and exacerbating co-morbidities and be aware the possible future use of genotyping to make statin therapy safer.

- Initially treatment is with aggressive fluid therapy and reversal of hyperkalaemia.

- Lipid lowering therapy after statin induced rhabdomyolysis must be pursued carefully.

\section{Competing interests None.}

Patient consent Obtained.

\section{REFERENCES}

1 Herring R, Caldwell G, Wade S. Rhabdomyolysis caused by an interaction of simvastatin and fusidic acid. BMJ Case Rep 2009

2 Saeed NTM, Azam M. Rhabdomyolysis secondary to interaction between atorvastatin and fusidic acid. BMJ Case Rep 2009.

3 Pasternak RC, Smith SCJrBairey-Merz CN, et al. ACC/AHA/NHLBI clinical advisory on the use and safety of statins. J Am Coll Cardiol 2002;40:567-72.

4 Hansen KE, Hildebrand JP, Ferguson EE, et al. Outcomes in 45 patients with statin-associated myopathy. Arch Intern Med 2005;165:2671-6.

5 Pedersen TR, Kjekshus J, Berg K, et al. Randomised Trial of Cholesterol Lowering in 4444 Patients with Coronary Heart Disease: The Scandinavian Simvastatin Survival Study (4S). Lancet 1994;344:1383-9.

6 BNF 59. British National Formulary, 2010

7 Law M, Rudnicka AR. Statin safety: a systematic review. Am J Cardiol 2006; 97:52C-60C.

8 Thompson PD, Clarkson P, Karas RH. Statin-associated myopathy. JAMA 2003;289:1681-90.

9 Graham DJ, Staffa JA, Shatin D, et al. Incidence of hospitalized rhabdomyolysis in patients treated with lipid-lowering drugs. JAMA 2004;292:2585-90.

10 Flint OP, Masters BA, Gregg RE, et al. Inhibition of cholesterol synthesis by squalene synthase inhibitors does not induce myotoxicity in vitro. Toxicol Appl Pharmacol 1997:145:91-8.

11 Phillips PS, Phillips CT, Sullivan MJ, et al. Statin myotoxicity is associated with changes in the cardiopulmonary function. Atherosclerosis 2004;177:183-8. 


\section{BMJ Case Reports}

12 Hsiang B, Zhu Y, Wang Z, et al. A novel human hepatic organic anion transporting polypeptide (OATP2). Identification of a liver-specific human organic anion transporting polypeptide and identification of rat and human hydroxymethylglutaryl-CoA reductase inhibitor transporters. J Biol Chem 1999;274:37161-8

13 Omar MA, Wilson JP, Cox TS. Rhabdomyolysis and HMG-CoA reductase inhibitors. Ann Pharmacother 2001;35:1096-107.

14 Schreiber DH, Anderson TR. Statin-induced rhabdomyolysis. J Emerg Med 2006;31:177-80.

15 Shek A, Ferrill MJ. Statin-fibrate combination therapy. Ann Pharmacother 2001;35:908-17.

16 Rosenberg AD, Neuwirth MG, Kagen LJ, et al. Intraoperative rhabdomyolysis in a patient receiving pravastatin, a 3-hydroxy-3-methylglutaryl coenzyme $\mathrm{A}$ (HMG CoA) reductase inhibitor. Anesth Analg 1995;81:1089-91.

17 Thompson PD, Zmuda JM, Domalik LJ, et al. Lovastatin increases exerciseinduced skeletal muscle injury. Metab Clin Exp 1997;46:1206-10.

18 Bottorff M, Hansten P. Long-term safety of hepatic hydroxymethyl glutaryl coenzyme A reductase inhibitors: the role of metabolism-monograph for physicians. Arch Intern Med 2000;160:2273-80.

19 Bolego C, Baetta R, Bellosta S, et al. Safety considerations for statins. Curr Opin Lipidol 2002:13:637-44.

20 Stefano B, Rodolfo P, Alberto C. Safety of statins: focus on clinical pharmacokinetics and drug interactions. Circulation 2004;109(Suppl III):III50-7.

21 Law MR, Wald NJ, Rudnicka AR. Quantifying effect of statins on low density lipoprotein cholesterol, ischaemic heart disease, and stroke: systematic review and meta-analysis. BMJ 2003:326:1423.
22 Prueksaritanont T, Subramanian R, Fang X, et al. Glucuronidation of statins in animals and humans: a novel mechanism of statin lactonization. Drug Metab Dispos 2002;30:505-12

23 Liao JK. Safety and efficacy of statins in Asians. Am J Cardiol 2007:99:410-14.

24 Sathasivam S, Lecky B. Statin induced myopathy. BMJ 2008;337:a2286.

25 SEARCH Collaborative Group, Link E, Parish S, et al. SLCO1B1 variants and statin-induced myopathy - a genomewide study. N Engl J Med 2008;359:789-99.

26 Ramrakha P, Moore K. Oxford Hanbook of Acute Medicine. Second edition. New York: Oxford University Press 2004:392-3.

27 Khan FY. Rhabdomyolysis: a review of the literature. Neth J Med 2009:67:272-83.

28 Bagley WH, Yang H, Shah KH. Rhabdomyolysis. Intern Emerg Med 2007;2:210-18.

29 Stuart W, Smellie A. Testing pitfalls and summary of guidance in lipid management. BMJ 2006;333:83-6.

30 O'Mahony C, Campbell VL, Al-Khayatt MS, et al. Rhabdomyolysis with atorvastatin and fusidic acid. Postgrad Med J 2008;84:325-7.

31 Antons KA, Williams CD, Baker SK, et al. Clinical perspectives of statininduced rhabdomyolysis. Am J Med 2006;119:400-9.

32 Vladutiu GD, Simmons Z, Isackson PJ, et al. Genetic risk factors associated with lipid-lowering drug-induced myopathies. Muscle Nerve 2006:34:153-62.

This pdf has been created automatically from the final edited text and images.

Copyright 2010 BMJ Publishing Group. All rights reserved. For permission to reuse any of this content visit

http://group.bmi.com/group/rights-licensing/permissions.

BMJ Case Report Fellows may re-use this article for personal use and teaching without any further permission.

Please cite this article as follows (you will need to access the article online to obtain the date of publication).

Patel BR, Choudhury M. Rhabdomyolysis with simvastatin. BMJ Case Reports 2010;10.1136/bcr.12.2009.2552, date of publication

Become a Fellow of BMJ Case Reports today and you can

- Submit as many cases as you like

- Enjoy fast sympathetic peer review and rapid publication of accepted articles

- Access all the published articles

- Re-use any of the published material for personal use and teaching without further permission

For information on Institutional Fellowships contact consortiasales@bmjgroup.com

Visit casereports.bmj.com for more articles like this and to become a Fellow 